\title{
The total artificial heart in pediatrics: outcomes in an evolving field
}

\author{
Chet R. Villa, Ryan A. Moore, David L. Morales, Angela Lorts \\ The Heart Institute, Cincinnati Children's Hospital Medical Center, Cincinnati, OH, USA \\ Correspondence to: Chet R. Villa, MD. 3333 Burnet Ave, MLC 2003, Cincinnati, OH 45229, USA. Email: Chet.villa@cchmc.org.
}

\begin{abstract}
Background: The use of the SynCardia temporary total artificial heart (TAH-t) in adults has increased with time. The development of the smaller, $50 \mathrm{cc}$ TAH-t has expanded the potential applications of the device in children. We sought to describe the evolving use of the TAH-t over time and describe outcomes in the current era.

Methods: The SynCardia database was queried to identify all pediatric patients $\leq 18$ years of age implanted with the device between December 1985 and October 2019. Patient demographics, clinical outcome and support characteristics collected.

Results: Fifty-one children were supported, 36 with the $70 \mathrm{cc}$ TAH-t and 15 with the $50 \mathrm{cc}$ TAH-t with a total support time of 6,243 days. The number of implants has increased with time (19 between 2015 and 2019). A total of 13 patients have been converted to Freedom Driver support, seven 50 cc TAH-t and six 70 cc TAH-t. The majority of implants in the last 5 years $(15 / 19,79 \%)$ have been with the 50 cc TAH-t. The most common diagnosis was dilated cardiomyopathy [24 (47\%)] and the average age at the time of implant was $16 \pm 2$ years old. Overall survival for the patient cohort was $71 \%$.

Conclusions: The use of the SynCardia TAH-t to support children with end-stage heart failure has increased over time. Clinical outcomes with both the $50 \mathrm{cc}$ and $70 \mathrm{cc}$ TAH-t are similar to reported outcomes in adults who require TAH-t or other methods of biventricular support.
\end{abstract}

Keywords: Total artificial heart; pediatrics; congenital heart disease

Submitted Jan 18, 2020. Accepted for publication Feb 19, 2020.

doi: $10.21037 /$ acs.2020.02.15

View this article at: http://dx.doi.org/10.21037/acs.2020.02.15

\section{Introduction}

The last 15 years have seen a rapid evolution in the field of pediatric mechanical circulatory support. The frequency of use of ventricular assist device (VAD) or temporary total artificial heart (TAH- $\mathrm{t}$ ) as a bridge to transplantation has more than doubled as almost one third of patients are bridged with a VAD or TAH (1). The numbers are even more dramatic for patients $>11$ years of age where more than half of patients are bridged to transplant on a VAD or TAH. While the majority of these patients have been supported with a VAD, recent advances in $3 \mathrm{D}$ virtual implantation and the development of the smaller, $50 \mathrm{cc}$ SynCardia TAH-t (SynCardia Systems, LLC, Tucson, Ariz) have expanded the use of the TAH in children and small adults $(2,3)$. In recent years, novel implantation methods have allowed the implantation of the device in patients with Fontan circulation and children as young as 10 years of age and as small as $0.9 \mathrm{~m}^{2}$ body surface area (BSA) (4-7).

Data regarding the use of the TAH-t in children is generally limited to individual case reports/case series. Given the evolving use of the device, this study sought to investigate the outcomes for pediatric patients supported with the TAH-t.

\section{Methods}

\section{Data collection and presentation}

SynCardia Systems, LLC retains records of all TAH- $t$ implantations including basic patient characteristics and clinical outcomes. The SynCardia database was queried to identify all pediatric patients $\leq 18$ years of age implanted 


\begin{tabular}{|c|c|c|}
\hline \multicolumn{3}{|l|}{ Age group (\%) } \\
\hline$\leq 11$ years old & $2(13.3)$ & $1(2.8)$ \\
\hline $12-18$ years old & $13(86.7)$ & $35(97.2)$ \\
\hline Age range (years) & $10-18$ & $9-18$ \\
\hline \multicolumn{3}{|l|}{$\operatorname{Sex}(\%)$} \\
\hline Male & $11(73.3)$ & $29(80.6)$ \\
\hline \multicolumn{3}{|l|}{ Diagnosis (\%) } \\
\hline Post heart transplant graft failure & $2(13.3)^{*}$ & $2(5.6)$ \\
\hline LVAD failure & $1(6.7)$ & $3(8.3)$ \\
\hline Hypertrophic cardiomyopathy & $1(6.7)$ & $2(5.6)$ \\
\hline Restrictive cardiomyopathy & $0(0.0)$ & $2(5.6)$ \\
\hline Valvular cardiomyopathy & $0(0.0)$ & $1(2.8)$ \\
\hline Unspecified & $2(13.3)$ & $1(2.8)$ \\
\hline
\end{tabular}

Results presented as number (frequency) or average \pm standard deviation. *, implanted sequentially after ventricular assist device in patient with graft dysfunction. TAH-t, temporary total artificial heart.

with the device between December 1985 and October 2019. The database contains a total of 1,876 patients. Three patients did not have a documented date of birth and were excluded. Interagency Registry for Mechanically Assisted Circulatory Support (INTERMACS) profile was collected beginning in 2014 and is reported for all patients for whom the data is available, this includes 9 patients implanted with a $50 \mathrm{cc}$ TAH-t and 2 patients with a $70 \mathrm{cc}$ TAH-t. BSA and T10 measurements (posterior sternum to anterior spine measurement at T10) were infrequently reported in the database on patients prior to 2014. Eleven patients (31\%) implanted with a $70 \mathrm{cc}$ TAH-t had BSA values recorded and $6(17 \%)$ patients had a T10 measurement available. Thus, the BSA and T10 measurements are reported for the $50 \mathrm{cc}$ devices alone.

Data was presented as average, mean \pm standard deviation, or median (range) as appropriate. Proportion (frequency) data is also provided as percentages. Statistical comparative tests were not performed.

\section{Results}

\section{Demographics and support duration}

Fifty-one patients were supported with the TAH-t during the study period (Table 1). Fifteen of the patients (29\%) were implanted with a $50 \mathrm{cc}$ TAH-t. There has been a progressive increase in the number of implants over time with a total of 19 implants between 2015 and 2019 (Figure 1). The majority (15 of 19, 79\%) of patients supported with a TAH-t in the last 5 years were implanted with the $50 \mathrm{cc}$ device.

The most common implant diagnosis for the $50 \mathrm{cc}$ and the $70 \mathrm{cc}$ was dilated cardiomyopathy (Table 1). The majority of patients implanted were 12-18 years of age, with 2 patients $<12$ years old implanted with a $50 \mathrm{cc}$ TAH-t and 1 patient $<12$ years-old implanted with a $70 \mathrm{cc}$ TAH-t. The median age for the $50 \mathrm{cc}$ and $70 \mathrm{cc}$ patients were 15 and 16 years respectively. Nine of the $50 \mathrm{cc}$ TAH-t patients had an INTERMACS profile recorded, 6 were INTERMACS 1 , 


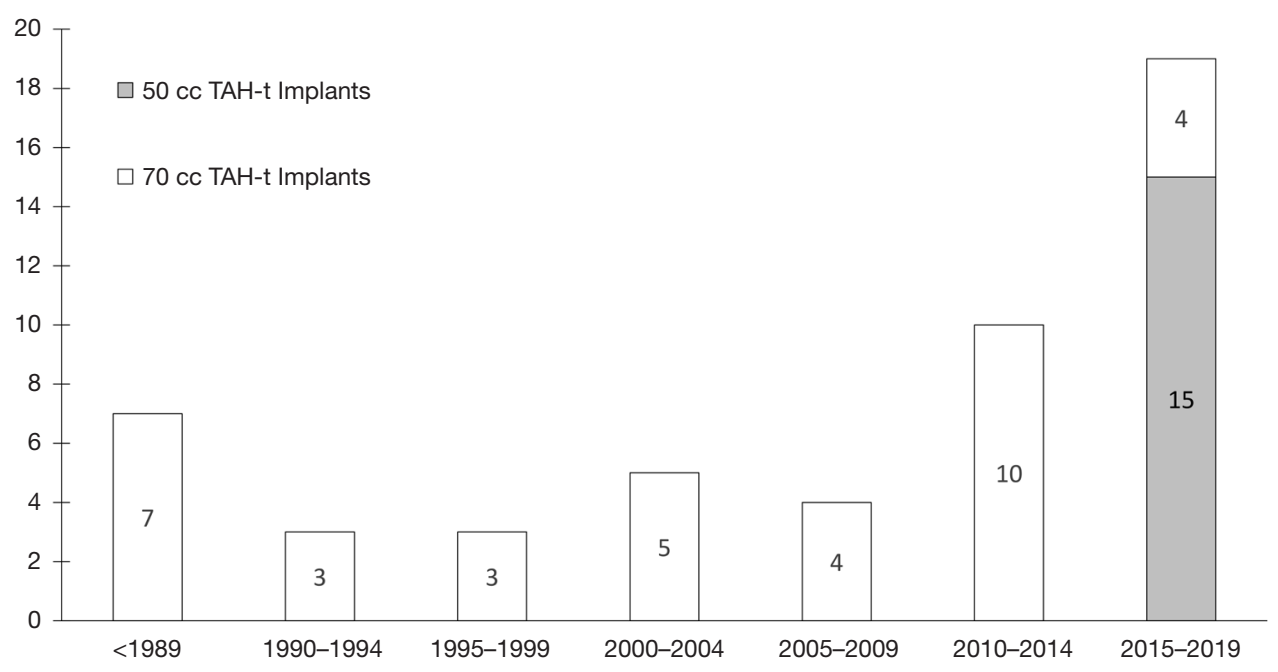

Figure 1 Number of pediatric TAH-t implants in 5-year increments stratified by device size.

\begin{tabular}{lll}
\hline \multicolumn{2}{l}{ Table 2 Support characteristics for pediatric patients ( $<19$ years) implanted with a TAH-t stratified by device size } \\
\hline Characteristics & $50 \mathrm{cc}$ TAH-t patients, N=15 & 70 cc TAH-t patients, N=36 \\
\hline $\begin{array}{ll}\text { Cumulative duration of support (days) } \\
\text { Average duration of support (days) }\end{array}$ & 2,171 & 4,072 \\
Duration of support (\%) & $145 \pm 165 \pm 204$ \\
$0-6$ months & $11(73.3)$ & $30(83.3)$ \\
$6-12$ months & $2(13.3)$ & $2(5.6)$ \\
$12-24$ months & $2(13.3)$ & $3(8.3)$ \\
$>24$ months & $0(0.0)$ & $1(2.8)$
\end{tabular}

2 patients were INTERMACS 2 and 1 was INTERMACS 4. Only two $70 \mathrm{cc}$ TAH-t patients had and INTERMACS profile recorded, 1 INTERMACS 1, and 1 INTERMACS 2 patient.

The patients have been supported for a total of 6,243 days. The average support duration was 145 days and 113 days for $50 \mathrm{cc}$ and $70 \mathrm{cc}$ TAH-t patients respectively. The majority of patients were supported for 6 months or less (Table 2).

\section{Patient size for $50 \mathrm{cc}$ TAH-t patients}

BSA data and T10 measurement were available for the majority of $50 \mathrm{cc}$ implant patients. Thirteen (87\%) of the $50 \mathrm{cc}$ patients had BSA values available and $10(67 \%)$ had
T10 measurements. The average BSA was $1.5 \mathrm{~m}^{2}$ and the smallest patient implanted had a BSA of $1.16 \mathrm{~m}^{2}$. The average T10 measurement was $11 \mathrm{~cm}$ with the shortest distance $7.9 \mathrm{~cm}$.

\section{Freedom driver support}

A total of 13 patients have been converted to Freedom Driver support, seven $50 \mathrm{cc}$ TAH-t patients and six $70 \mathrm{cc}$ TAH-t patients (Table 3). The patients were converted to the Freedom Driver at an average of 68 and 83 days respectively. A total of 8 patients were discharged from the hospital, three $50 \mathrm{cc}$ TAH-t patients and five $70 \mathrm{cc}$ TAH-t patients. Twelve (92\%) of the patients converted to the Freedom Driver have been transplanted and 1 is currently 


\begin{tabular}{|c|c|c|}
\hline \multicolumn{3}{|l|}{ Freedom driver support } \\
\hline Cumulative duration of freedom driver support (days) & 1,176 & 1,613 \\
\hline Average duration of freedom driver support (days) & $168 \pm 156$ & $269 \pm 362$ \\
\hline \multicolumn{3}{|l|}{ Discharge on freedom driver } \\
\hline Number of patients discharged (\%) & $3(42.9)$ & $5(83.3)$ \\
\hline Duration from implant to discharge (days) & $170 \pm 113$ & $127 \pm 109$ \\
\hline \multicolumn{3}{|l|}{ Outcome on freedom driver support (\%) } \\
\hline Death & $0(0.0)$ & $0(0.0)$ \\
\hline
\end{tabular}

Results presented as average \pm standard deviation and number (frequency). TAH-t, temporary total artificial heart.

\begin{tabular}{lll}
\multicolumn{2}{l}{ Table 4 Clinical outcomes for pediatric patients (<19 years) supported with a TAH-t stratified by device size } \\
\hline Clinical outcomes & 50 cc TAH-t patients, $\mathrm{N}=15$ & 70 cc TAH-t patients, N=36 \\
\hline $\begin{array}{l}\text { Overall clinical outcome (\%) } \\
\text { Transplant }\end{array}$ & $11(73.3)$ & $24(66.7)$ \\
Alive on device & $1(6.7)$ & $0(0.0)$ \\
Died on device & $3(20.0)$ & $12(33.3)$ \\
60-day clinical outcome (\%) & $13(36.1)$ \\
Transplant & $5(33.3)$ & $14(38.9)$ \\
Alive on device & $10(66.7)$ & $9(25.0)$ \\
Death & $0(0.0)$ &
\end{tabular}

on support.

\section{Outcomes}

Overall survival (alive on device or transplanted) is $71 \%$ [36] with a total of 35 patients being successfully supported to transplantation and a single patient currently being supported (Table 4). All of the patients supported with the $50 \mathrm{cc}$ TAH-t underwent heart transplantation or were alive on device at 60 days. The three patients who did not survive on the $50 \mathrm{cc}$ TAH-t passed away at day 62,103 , and 110 , respectively. Twenty-seven ( $75 \%)$ of the patients supported with the $70 \mathrm{cc}$ TAH-t underwent heart transplantation or were alive on device at 60 days.

\section{Discussion}

The current report describes the evolving current outcomes and evolving use of the TAH-t in children. There has been an appreciable increase in the number of children implanted with the device in the last 5 years and a shift toward greater use of the $50 \mathrm{cc}$ TAH-t. The current report also documents the feasibility of hospital discharge and implantation of the device in children as young as 9 years of age. These data are 
encouraging and suggest this device is an effective support strategy in selected children requiring biventricular support.

Historically, the use of the TAH-t was limited to patients with a $B S A \geq 1.7 \mathrm{~m}^{2}$ and an anterior-posterior distance from the sternum to the $10^{\text {th }}$ thoracic vertebra of at least $10 \mathrm{~cm}(8)$. This limited use of the device in children and young adults (9). As pediatric centers have garnered experience with the device and with mechanical circulatory support in general, the use of device has increased. Increased application of TAH-t at individual centers has been driven by extending the lower limits of "acceptable" patient size through 3D modeling, virtual implantation and novel implantation approaches to children with congenital heart disease $(3,5,6)$. Multicenter collaboration using these techniques have allowed centers to implant children as small as $0.9 \mathrm{~m}^{2}$ BSA and with a variety of anatomic configurations. While these trends increased the number of device implants, it was unclear if a more aggressive approach to implantation would come at the expense of clinical outcomes. The current report suggests good clinical outcomes in the current era are possible using this approach as these are patients who do not have good VAD support options. The TAH does not supplant the use of VAD therapy in programs but rather provides a possible solution for those patients in whom VAD therapy has been shown not to be reliable or effective. It is especially notable that the clinical outcomes with the $50 \mathrm{cc}$ TAH-t are similar to other reports documenting the clinical outcomes for adult patients requiring biventricular support and for adults receiving a total artificial heart. A recent analysis of the EUROMACS registry reported a $67 \%$ survival rate at 3 months for adults who received primary biventricular support (10), while INTERMACS reported a 3-month survival of $73 \%$ for adults implanted with a total artificial heart (11).

The current report is also notable for documenting good clinical outcomes and the potential for discharge on the Freedom Driver. While the number of patient discharges is small ( 8 patients), the frequency of discharge $(61 \%)$ is notable when considering the total number of patients who were converted to the Freedom Driver was also limited. The frequency of discharge is also similar to the reported frequency of pediatric patients discharged with a continuous flow VAD in the current era (55\%) (12). The relatively low frequency of discharge compared to adults is likely multifactorial, including the relatively short waitlist time for adolescents in the United States, and evolving experience with hospital discharge with children on VAD/TAH-t.
It was not possible to assess the reasons for continued hospitalization in the group of children who were never discharged given the limits of the database. There are ongoing projects analyzing the barriers to discharge within the field that may help to elucidate reasons for ongoing hospitalization. The most notable is a multicenter project through the Advanced Cardiac Therapies Improving Outcomes Network (ACTION) focused on facilitating hospital discharge in children supported with a VAD or TAH. This network will also collect patient level data and clinical management data to further understand the predictors of clinical outcome and work to improve clinical outcomes in a rare patient population with severe disease.

\section{Limitations}

Patient level data including the specifics of diagnosis including the severity and nature of congenital heart disease and patient condition (e.g., end organ dysfunction) are not available in the clinical database. The specifics of patient size including thoracic dimensions and body size have only been collected since 2014 and remain incomplete. This lack of granular data limits the ability to understand the impact of size and complex congenital heart disease on clinical outcomes.

\section{Conclusions}

There has been an increase in the number of the children supported with TAH-t over time, which has coincided with the development of the $50 \mathrm{cc}$ TAH-t. The clinical outcomes to date suggest both the $50 \mathrm{cc}$ and $70 \mathrm{cc}$ TAH-t are an effective supportive strategy in high acuity patients requiring biventricular support. Multicenter collaboration will be integral to improving outcomes especially among the smallest patients and those with congenital heart disease, which may require novel surgical implant techniques.

\section{Acknowledgement}

The authors express their gratitude and deep appreciation to the Cincinnati Children's Media Lab Team for creating the SynCardia Animation. The Media Lab includes Director Ken Tegtmeyer MD, Associate Director Ryan A. Moore MD, Lead Animator Jeff Cimprich BFA, Animator Cat Musgrove BFA, and Animator Matt Nelson BFA. Previous Media Lab Animator Ren Wilkey BFA also contributed to this project. Peace Madueme MD provided voiceover 
work. David Morales MD provided extensive support and significant contributions to the vision and content creation of this animation. This work was made possible by the Jr. Co-Op Foundation Grant that Dr. Morales and Dr. Moore received to create surgical animations for provider, patient, and family education.

\section{Footnote}

Conflicts of Interest: Dr. Lorts is a consultant for SynCardia, Abbott, Medtronic, and Berlin Heart. Dr. Morales is a consultant for Cormatrix/Azyio, Berlin Heart, SynCardia, Abbott, Medtronic and instructor for SynCardia and Berlin Heart. The other authors have no conflicts of interest to declare.

\section{References}

1. Rossano JW, Singh TP, Cherikh WS, et al. The International Thoracic Organ Transplant Registry of the International Society for Heart and Lung Transplantation: Twenty-second pediatric heart transplantation report 2019; Focus theme: Donor and recipient size match. J Heart Lung Transplant 2019;38:1028-41.

2. Wells D, Villa CR, Simón Morales DL. The 50/50 cc Total Artificial Heart Trial: Extending the Benefits of the Total Artificial Heart to Underserved Populations. Semin Thorac Cardiovasc Surg Pediatr Card Surg Annu 2017. doi: 10.1053/j.pcsu.2016.09.004.

3. Moore RA, Lorts A, Madueme PC, et al. Virtual implantation of the $50 \mathrm{cc}$ SynCardia total artificial heart. J Heart Lung Transplant 2016;35:824-7.

Cite this article as: Villa CR, Moore RA, Morales DL, Lorts A. The total artificial heart in pediatrics: outcomes in an evolving field. Ann Cardiothorac Surg 2020;9(2):104-109. doi: 10.21037/ acs.2020.02.15
4. Beasley GS, Allen K, Pahl E, et al. Successful Bridge to Transplant in a Pediatric Patient Using the SynCardia 50 cc Total Artificial Heart. ASAIO J 2020;66:e33-5.

5. Rossano JW, Goldberg DJ, Fuller S, et al. Successful use of the total artificial heart in the failing Fontan circulation. Ann Thorac Surg 2014;97:1438-40.

6. Woods RK, Niebler R, Kindel S, et al. A new method for implanting a total artifical heart in the patient with a Fontan circulation. J Thorac Cardiovasc Surg 2019;157:353-5.

7. Alaeddine M, Ploutz M, Arabía FA, et al. Implantation of total artificial heart in a 10-year-old after support with a temporary perventricular assist device. J Thorac Cardiovasc Surg 2020;159:e227-9.

8. Copeland JG, Smith RG, Arabia FA, et al. Cardiac replacement with a total artificial heart as a bridge to transplantation. N Engl J Med 2004;351:859-67.

9. Morales DLS, Lorts A, Rizwan R, et al. Worldwide Experience with the Syncardia Total Artificial Heart in the Pediatric Population. ASAIO J 2017;63:518-9.

10. Vierecke J, Gahl B, de By TMMH, et al. Results of primary biventricular support: an analysis of data from the EUROMACS registry. Eur J Cardiothorac Surg 2019;56:1037-45.

11. Arabía FA, Cantor RS, Koehl DA, et al. Interagency registry for mechanically assisted circulatory support report on the total artificial heart. J Heart Lung Transplant 2018;37:1304-12.

12. Conway J, Miera O, Adachi I, et al. Worldwide Experience of a Durable Centrifugal Flow Pump in Pediatric Patients. Semin Thorac Cardiovasc Surg 2018;30:327-35. 